\title{
Fasting Plasma Glucose Variability in Midlife and the Risk of Parkinson's Disease: A Nationwide Population- Based Study
}

Hye Soo Chung

Hallym University

Ji Sung Lee

Asan Medical Center

Jung A Kim

Korea University

Eun Roh

Korea University

You-Bin Lee

Korea University

So-Hyeon Hong

Korea University

Hye Jin Yoo

Korea University

Sei Hyun Baik

Korea University

Nan Hee Kim

Korea University

Ji A Seo

Korea University

Ji Hee Yu

Korea University

Sin Gon Kim

Korea University

Nam Hoon Kim

Korea University

Kyung Mook Choi ( $\nabla$ medica7@gmail.com )

Korea University https://orcid.org/0000-0001-6175-0225

Original investigation

Keywords: glycemic variability, visit-to-visit, fasting plasma glucose, Parkinson's disease, midlife

Posted Date: March 2nd, 2020 
DOI: https://doi.org/10.21203/rs.3.rs-15545/v1

License: (c) (i) This work is licensed under a Creative Commons Attribution 4.0 International License. Read Full License

Version of Record: A version of this preprint was published at Diabetes \& Metabolism on May 1st, 2021. See the published version at https://doi.org/10.1016/j.diabet.2020.08.006. 


\section{Abstract}

Background - Athough an association exists between type 2 diabetes and Parkinson's disease (PD), the implications of glycemic variability on PD are unknown. We assessed the future risk of incident PD according to visit-to-visit fasting plasma glucose (FPG) variability; this was calculated using standard deviation (FPG-SD), coefficient variance (FPG-CV), and variability independent of the mean (FPG-VIM).

Methods - Using the Korean National Health Insurance Service-Health Screening Cohort, we followed 131,625 Korean adults without diabetes. This study population was divided into a midlife group ( $<65$ years) and an elderly group ( $\geq 65$ years), during a median follow-up of 8.4 years.

Results - The adjusted hazard ratios (HRs) were calculated using a multivariable Cox proportional hazard analysis. In the midlife group, the HRs for incident PD in the highest quartile of FPG variability, as measured using SD, CV, and VIM, were 1.35 (95\% confidence interval (Cl), 1.07-1.70), 1.31 (95\% Cl, 1.04-1.65), and 1.33 $(95 \% \mathrm{Cl}, 1.06-1.67)$, respectively, when compared to the lowest quartile group. However, the incident PD was not different depending on FPG variability in the elderly group. Kaplan-Meier curves of PD probability showed a progressively increasing risk of PD according to the higher FPG variability in the midlife group. According to a multivariable adjusted model, a 1-SD unit increment in glycemic variability was associated with a $9 \%$ higher risk for incident PD in the midlife group.

Conclusions - Increased long-term glycemic variability is a preceding risk factor for developing PD in the midlife population without diabetes.

\section{Background}

Parkinson's disease (PD) is a common neurodegenerative disease, occurring generally in $1-2 \%$ of the global population of people over the age of 65 years [1]. PD is a progressive disorder caused by the loss of the dopaminergic neurons in the pars compacta, a portion of the substantia nigra, located in striatum [1]. The effects of the reduction of dopamine are suggested to be caused by oxidative stress, chronic inflammation, mitochondrial dysfunction, deposition of abnormal protein, and systemic and central insulin resistance, which are shared pathophysiologic mechanisms between PD and type 2 diabetes [1].

Previous studies have reported that patients with type 2 diabetes have an increased risk of developing PD. In a prospective study including 51,552 Finnish adults, a multivariate-adjusted association between type 2 diabetes and PD was found [2]. In the National Institutes of Health (NIH)-American Association of Retired Persons (AARP) Diet and Health Study of the U.S., the type 2 diabetes group had a $41 \%$ higher risk of PD than the group without diabetes $(\mathrm{OR}=1.41 ; 95 \% \mathrm{Cl}=1.20-1.66)$ [3]. Moreover, the risk of $\mathrm{PD}$ abruptly increased among patients with type 2 diabetes who have had it for 10 years or more compared to those who have had it for less than 10 years $(\mathrm{OR}=1.75 ; 95 \% \mathrm{Cl}=1.36-2.25)$ [3]. In Taiwan's large population cohort, individuals with diabetes showed a much higher risk of developing PD than those without diabetes [4]. Furthermore, in a prospective study of male physicians in the U.S., subjects with type 2 diabetes had a 34\% increased risk of incident PD over 23 years compared to those without type 2 diabetes before and after adjusting for other compounding factors [5]. Using the nationwide Danish Hospital Register data, Schernhammer et al. reported that diabetes was associated with an increased risk of PD; this risk was even greater for early-onset PD [6]. Moreover, diabetes accelerated the aggravation of both cognitive and motor signs in patients with PD [7]. A large portion of PD patients showed 
abnormal glucose tolerance or hyperglycemia [8]. Furthermore, Moral et al. reported genetic links between type 2 diabetes and PD using a whole genome transcriptome profile [9]. It was not clarified whether this relationship is due to sustained hyperglycemia, an event of hypoglycemia, the use of diabetes medication, or glycemic variability.

Recently, some studies showed that increased glycemic variability or intermittent high glucose levels made greater contributions to oxidative stress, inflammatory cytokines, endothelial dysfunction, and insulin resistance compared to chronic hyperglycemia $[10,11]$. Previously, it had been demonstrated that increased long-term glycemic variability is significantly associated with microvascular and macrovascular events [12-14] and allcause mortality in patients with type 2 diabetes [15]. We reported that visit-to-visit glycemic variability predicted the future risk of type 2 diabetes in the Korean population without diabetes [16]. According to these results, it is suggested that long-term glycemic variability may be a valuable predictor for chronic diseases and mortality beyond mean glucose levels [17].

Therefore, we hypothesized that long-term glycemic variability and PD are intimately related due to their shared mechanistic pathophysiology. Nevertheless, to the best of our knowledge, no previous studies have showed the relationship between long-term glucose variability and incident PD. Our study was based on the longitudinal National Health Insurance Service-National Health Screening Cohort (NHIS-HEALS) database in Korea. Using this data, we examined the association between visit-to-visit variability of FPG and the risk of developing PD in the general population without diabetes.

\section{Methods}

\section{Study participants}

The NHIS of South Korea is a government-managed mandatory public health insurance program that covers approximately 50 million Korean residents (almost $98 \%$ of the entire population). Under this program, it is recommended to have a general health examination at least biannually [18]. The NHIS includes sociodemographic information (e.g., age, sex, and income), a health examination database (e.g., standardized self-reported questionnaires on physical activity, smoking status, and alcohol consumption; anthropometric measurements; blood pressure; and laboratory tests), and claim databases of outpatient and inpatient records (e.g., diagnoses, pharmacy, and death). Anthropometric measurements (e.g., height and weight) and laboratory tests were done after an overnight fast, and quality control procedures were confirmed by the Korean Association of Laboratory Quality Control. The NHIS-HEALS database was randomly selected to include almost $10 \%$ of the entire population between 40 and 79 years old within the NHIS database, who had taken the national health examination. The sample initially collected 206,661 people who had taken the examination in 2007 (index year). After excluding individuals who had undergone one or two health examinations from January 1, 2002 to December 31, 2007 ( $n=30,954)$, individuals who had missing data for at least one variable $(n=15,301)$, and individuals who had a previous diagnosis of diabetes and PD prior to 2007 ( $n=28,781)$. Finally, a total of 131,625 individuals were included in the analysis (Supplemental Fig. 1). During the median follow-up of 8.4 years (Interquartile range: 8.2, 8.6) in the entire cohort, 1,263 individuals developed PD (Age < 65: 554, Age $\geq 65:$ 709). These protocols were approved by the NHIS review committee. The Korea University institutional review board (IRB) approved the study protocol in accordance with the Declaration of Helsinki of the World Medical 
Association. Informed consent was waived because anonymous and de-identified information was used for the analysis.

\section{Definition of FPG variability}

The FPG variability was calculated using three or more FPG values measured during the participants' health examinations that were completed between January 1, 2002 to December 31, 2007. The following quantities of FPG value measurements per participant were included: 3 measurements $(n=67120 ; 51.0 \%), 4$ measurements ( $n$ $=14075 ; 10.7 \%), 5$ measurements $(n=18794 ; 14.3 \%)$, and 6 measurements $(n=31636 ; 24.0 \%)$. We used three indices for prescriptive FPG variability: standard deviation (FPG-SD), coefficient of variation (FPG-CV), and variability independent of the mean (FPG-VIM). The CV was defined as SD/mean $\times 100 \%$ and VIM was defined as $100 \times S D /$ Mean $\beta$, where $\beta$ is the regression coefficient, based on the natural logarithm of the SD divided by the natural logarithm of the mean.

\section{Measurements and definitions}

We examined newly occurring PD as primary outcomes from January 1, 2008 to December 31, 2015. The diagnosis of PD was defined using the International Classification of Diseases, Tenth Revision (ICD-10) code G20. During hospitalisation, patients with one or more diagnoses of G20 codes and during outpatient clinic visits, those with G20 codes having been recorded at least twice were considered to have PD [19, 20]. The presence of diabetes was defined based on the criteria of a fasting glucose level $\geq 7.0 \mathrm{mmol} / \mathrm{L}$ or by having at least one prescription claim per year for antidiabetic medication under the ICD-10 codes E10-E14. The presence of hypertension was defined based on the criteria of systolic/diastolic blood pressure $\geq 140 / 90 \mathrm{mmHg}$ or by having at least one prescription claim per year for an antihypertensive agent under ICD-10 codes I10-I15. The presence of dyslipidemia was defined based on the criteria of total cholesterol $\geq 6.2 \mathrm{mmol} / \mathrm{L}$ or by having at least one prescription claim per year for an anti-hyperlipidemic agent under ICD-10 code E78. The diagnosis of stroke was defined as ICD-10 codes I60-164 on the admission record with computerized tomography or magnetic resonance imaging claim data. The diagnosis of chronic kidney disease (CKD) was defined as ICD-10 codes N18 or N19. Body mass index (BMI) was calculated as weight in kilograms divided by the square of height in meters $\left(\mathrm{kg} / \mathrm{m}^{2}\right)$. Smoking status and information concerning alcohol consumption were obtained from a questionnaire undertaken during the health examination. Regular exercise was defined as strenuous physical activity for at least 20 minutes, $\geq 5$ times/week. Income levels were dichotomized at the lower $10 \%$.

\section{Statistical analysis}

The baseline characteristics are presented as the mean \pm standard deviation for continuous variables or percentages for categorical variables. The participants were divided by age 65 years at the index year (2007). Then, they were classified into quartile groups according to the FPG variability value. Any differences between the groups were identified using the analysis of variance (ANOVA) for continuous variables or the $\chi^{2}$-test for categorical variables. Kaplan-Meier curves for the probability of PD were obtained for the four groups which were classified and expressed as quartiles of FPG variability. Hazard ratios (HRs) and 95\% confidence interval (CI) values for PD incidence were analyzed using Cox proportional hazards models for quartile groups of FPG variability. This was adjusted for age, sex, BMI, hypertension, dyslipidemia, smoking status, alcohol consumption, regular exercise, income, history of stroke, history of CKD, and mean FPG. We tested the assumption of proportionality of hazards using the numerical method proposed by Lin et al., derived from the cumulative sums of martingale-based residuals [21]. We found no evidence that violates the proportional hazards assumption. We 
used multivariable linear regression for the risk of $\mathrm{PD}$ to evaluate the association of a 1-SD increment for continuous FPG-SD, FPG-CV, and FPG-VIM after adjusting compounding factors. Especially, to estimate whether the FPG variability to PD occurrence association is independent for other risk factors, we analyzed with stepwise models adjusting for: 1) age and sex; 2) age, sex, BMI, hypertension, dyslipidemia, smoking status, alcohol intake, regular exercise, and income; 3 ) age, sex, body mass index, hypertension, dyslipidemia, smoking status, alcohol intake, regular exercise, income, history of stroke, and history of CKD; 4) age, sex, body mass index, hypertension, dyslipidemia, smoking status, alcohol intake, regular exercise, income, history of stroke, history of CKD, and mean FPG. All of the statistical results were analyzed using SAS 9.4 (SAS Institute Inc., Cary, NC, USA). A P-value $<0.05$ was assumed to indicate statistical significance. All the statistical analyses were performed by an experienced professional statistician, who was also one of authors (J.S.L.).

\section{Results}

\section{Baseline characteristics of the study population}

Table 1 presented the general characteristics of the study participants according to the quartiles of SD for FPG variability in individuals $<65$ years (midlife group) and those $\geq 65$ years (elderly group). The participants in the higher quartile of FPG-SD were older and heavier, more likely to be male, and had less healthy behaviors including smoking, alcohol consumption, and lack of exercise than the lower quartile group. In the midlife group, higher FPG variability was also incrementally associated with blood pressure, total cholesterol level, and the prevalence of hypertension and dyslipidemia. However, there were no significant increases in systolic blood pressure, total cholesterol level, or the prevalence of dyslipidemia according to the quartiles of FPG variability in the elderly group. In addition, FPG variability was not associated with the history of stroke or the history of CKD in either age groups. Similar patterns of characteristics among the study population were shown according to the quartiles of FPG-CV (Supplemental Table 1) and FPG-VIM (Supplemental Table 2). 
Table 1

Baseline characteristics of participants according to the fasting plasma glucose variability (SD)

\begin{tabular}{|c|c|c|c|c|c|c|c|c|c|c|}
\hline & \multicolumn{5}{|c|}{ Midlife (Age < 65 years) } & \multicolumn{5}{|c|}{ Elderly (Age $\geq 65$ years) } \\
\hline & Q1 & Q2 & Q3 & Q4 & $\begin{array}{l}\text { P- } \\
\text { value }\end{array}$ & Q1 & Q2 & Q3 & Q4 & $\begin{array}{l}\mathrm{P} \text { - } \\
\text { value }\end{array}$ \\
\hline $\begin{array}{l}\text { FPG-SD } \\
(\mathrm{mmol} / \mathrm{L})\end{array}$ & $\begin{array}{l}3.84 \\
\pm 1.28\end{array}$ & $\begin{array}{l}6.98 \\
\pm 0.77\end{array}$ & $\begin{array}{l}9.88 \\
\pm 0.94\end{array}$ & $\begin{array}{l}15.03 \\
\pm 2.96\end{array}$ & $\begin{array}{l}< \\
0.001\end{array}$ & $\begin{array}{l}3.48 \\
\pm 1.28\end{array}$ & $\begin{array}{l}7.01 \\
\pm 0.92\end{array}$ & $\begin{array}{l}10.36 \\
\pm 1.08\end{array}$ & $\begin{array}{l}16.37 \\
\pm 3.38\end{array}$ & $\begin{array}{l}< \\
0.001\end{array}$ \\
\hline $\mathrm{N}$ & 28087 & 28076 & 28092 & 28092 & & 4705 & 4934 & 4818 & 4821 & \\
\hline Age (years) & $\begin{array}{l}52.7 \\
\pm 5.5\end{array}$ & $\begin{array}{l}52.3 \\
\pm 5.4\end{array}$ & $\begin{array}{l}52.4 \\
\pm 5.5\end{array}$ & $\begin{array}{l}52.9 \\
\pm 5.6\end{array}$ & $\begin{array}{l}< \\
0.001\end{array}$ & $\begin{array}{l}70.9 \\
\pm 3.3\end{array}$ & $\begin{array}{l}70.8 \\
\pm 3.3\end{array}$ & $\begin{array}{l}70.8 \\
\pm 3.4\end{array}$ & $\begin{array}{l}71.0 \\
\pm 3.5\end{array}$ & 0.003 \\
\hline $\begin{array}{l}\text { Sex (male) (n, } \\
\%)\end{array}$ & $\begin{array}{l}13773 \\
(49.0)\end{array}$ & $\begin{array}{l}16117 \\
(57.4)\end{array}$ & $\begin{array}{l}17056 \\
(60.7)\end{array}$ & $\begin{array}{l}17894 \\
(63.7)\end{array}$ & $\begin{array}{l}< \\
0.001\end{array}$ & $\begin{array}{l}2269 \\
(48.2)\end{array}$ & $\begin{array}{l}2464 \\
(49.9)\end{array}$ & $\begin{array}{l}2534 \\
(52.6)\end{array}$ & $\begin{array}{l}2578 \\
(53.5)\end{array}$ & $\begin{array}{l}< \\
0.001\end{array}$ \\
\hline $\mathrm{BMI}\left(\mathrm{kg} / \mathrm{m}^{2}\right)$ & $\begin{array}{l}23.8 \\
\pm 2.7\end{array}$ & $\begin{array}{l}23.9 \\
\pm 2.8\end{array}$ & $\begin{array}{l}23.9 \\
\pm 2.8\end{array}$ & $\begin{array}{l}24.0 \\
\pm 2.8\end{array}$ & $\begin{array}{l}< \\
0.001\end{array}$ & $\begin{array}{l}23.6 \\
\pm 3.0\end{array}$ & $\begin{array}{l}23.6 \\
\pm 3.0\end{array}$ & $\begin{array}{l}23.8 \\
\pm 3.0\end{array}$ & $\begin{array}{l}23.6 \\
\pm 3.1\end{array}$ & 0.008 \\
\hline $\begin{array}{l}\text { Systolic BP } \\
(\mathrm{mmHg})\end{array}$ & $\begin{array}{l}122.6 \\
\pm 14.9\end{array}$ & $\begin{array}{l}123.2 \\
\pm 14.9\end{array}$ & $\begin{array}{l}124.0 \\
\pm 15.0\end{array}$ & $\begin{array}{l}125.2 \\
\pm 15.1\end{array}$ & $\begin{array}{l}< \\
0.001\end{array}$ & $\begin{array}{l}130.8 \\
\pm 16.7\end{array}$ & $\begin{array}{l}130.6 \\
\pm 16.7\end{array}$ & $\begin{array}{l}131.0 \\
\pm 16.5\end{array}$ & $\begin{array}{l}131.1 \\
\pm 17.2\end{array}$ & 0.364 \\
\hline $\begin{array}{l}\text { Diastolic BP } \\
(\mathrm{mmHg})\end{array}$ & $\begin{array}{l}76.9 \\
\pm 10.0\end{array}$ & $\begin{array}{l}77.5 \\
\pm 10.1\end{array}$ & $\begin{array}{l}78.0 \\
\pm 10.1\end{array}$ & $\begin{array}{l}78.7 \\
\pm 10.1\end{array}$ & $\begin{array}{l}< \\
0.001\end{array}$ & $\begin{array}{l}78.6 \\
\pm 10.3\end{array}$ & $\begin{array}{l}78.8 \\
\pm 10.2\end{array}$ & $\begin{array}{l}79.4 \\
\pm 10.2\end{array}$ & $\begin{array}{l}79.5 \\
\pm 10.4\end{array}$ & $\begin{array}{l}< \\
0.001\end{array}$ \\
\hline AST (IU/L) & $\begin{array}{l}25.1 \\
\pm 14.2\end{array}$ & $\begin{array}{l}25.4 \\
\pm 13.5\end{array}$ & $\begin{array}{l}25.7 \\
\pm 12.9\end{array}$ & $\begin{array}{l}26.7 \\
\pm 17.3\end{array}$ & $\begin{array}{l}< \\
0.001\end{array}$ & $\begin{array}{l}25.7 \\
\pm 11.8\end{array}$ & $\begin{array}{l}26.3 \\
\pm 14.1\end{array}$ & $\begin{array}{l}26.6 \\
\pm 13.1\end{array}$ & $\begin{array}{l}26.9 \\
\pm 14.5\end{array}$ & $\begin{array}{l}< \\
0.001\end{array}$ \\
\hline ALT (IU/L) & $\begin{array}{l}24.1 \\
\pm 19.0\end{array}$ & $\begin{array}{l}24.7 \\
\pm 18.8\end{array}$ & $\begin{array}{l}25.1 \\
\pm 18.7\end{array}$ & $\begin{array}{l}26.0 \\
\pm 20.3\end{array}$ & $\begin{array}{l}< \\
0.001\end{array}$ & $\begin{array}{l}21.2 \\
\pm 12.6\end{array}$ & $\begin{array}{l}21.9 \\
\pm 15.5\end{array}$ & $\begin{array}{l}22.1 \\
\pm 14.3\end{array}$ & $\begin{array}{l}22.2 \\
\pm 12.6\end{array}$ & 0.001 \\
\hline GGT (IU/L) & $\begin{array}{l}32.2 \\
\pm 37.4\end{array}$ & $\begin{array}{l}35.4 \\
\pm 42.6\end{array}$ & $\begin{array}{l}37.8 \\
\pm 46.3\end{array}$ & $\begin{array}{l}40.9 \\
\pm 50.8\end{array}$ & $\begin{array}{l}< \\
0.001\end{array}$ & $\begin{array}{l}29.1 \\
\pm 37.8\end{array}$ & $\begin{array}{l}30.9 \\
\pm 42.4\end{array}$ & $\begin{array}{l}31.9 \\
\pm 37.4\end{array}$ & $\begin{array}{l}34.0 \\
\pm 48.3\end{array}$ & $\begin{array}{l}< \\
0.001\end{array}$ \\
\hline $\begin{array}{l}\text { Total } \\
\text { cholesterol } \\
(\mathrm{mg} / \mathrm{dL})\end{array}$ & $\begin{array}{l}198.1 \\
\pm 35.1\end{array}$ & $\begin{array}{l}198.2 \\
\pm 35.2\end{array}$ & $\begin{array}{l}198.9 \\
\pm 35.8\end{array}$ & $\begin{array}{l}199.5 \\
\pm 36.3\end{array}$ & $\begin{array}{l}< \\
0.001\end{array}$ & $\begin{array}{r}197.9 \\
\pm 36.9\end{array}$ & $\begin{array}{l}197.9 \\
\pm 37.1\end{array}$ & $\begin{array}{l}198.4 \\
\pm 37.7\end{array}$ & $\begin{array}{l}197.8 \\
\pm 38.1\end{array}$ & 0.861 \\
\hline $\begin{array}{l}\text { Mean FPG } \\
(\mathrm{mmol} / \mathrm{L})\end{array}$ & $\begin{array}{l}90.3 \\
\pm 8.6\end{array}$ & $\begin{array}{l}90.5 \\
\pm 8.4\end{array}$ & $\begin{array}{l}91.1 \\
\pm 8.0\end{array}$ & $\begin{array}{l}92.6 \\
\pm 7.2\end{array}$ & $\begin{array}{l}< \\
0.001\end{array}$ & $\begin{array}{l}91.4 \\
\pm 9.2\end{array}$ & $\begin{array}{l}91.7 \\
\pm 8.9\end{array}$ & $\begin{array}{l}92.4 \\
\pm 8.6\end{array}$ & $\begin{array}{l}93.5 \\
\pm 7.5\end{array}$ & $\begin{array}{l}< \\
0.001\end{array}$ \\
\hline $\begin{array}{l}\text { Smoking } \\
\text { status (n, \%) }\end{array}$ & & & & & $\begin{array}{l}< \\
0.001\end{array}$ & & & & & $\begin{array}{l}< \\
0.001\end{array}$ \\
\hline Non-smoker & $\begin{array}{l}20797 \\
(74.0)\end{array}$ & $\begin{array}{l}19577 \\
(69.7)\end{array}$ & $\begin{array}{l}19087 \\
(67.9)\end{array}$ & $\begin{array}{l}18568 \\
(66.1)\end{array}$ & & $\begin{array}{l}3899 \\
(82.9)\end{array}$ & $\begin{array}{l}4095 \\
(83.0)\end{array}$ & $\begin{array}{l}3917 \\
(81.3)\end{array}$ & $\begin{array}{l}3846 \\
(79.8)\end{array}$ & \\
\hline Ex-smoker & $\begin{array}{l}2697 \\
(9.6)\end{array}$ & $\begin{array}{l}2988 \\
(10.6)\end{array}$ & $\begin{array}{l}2887 \\
(10.3)\end{array}$ & $\begin{array}{l}2692 \\
(9.6)\end{array}$ & & $\begin{array}{l}320 \\
(6.8)\end{array}$ & $\begin{array}{l}296 \\
(6.0)\end{array}$ & $\begin{array}{l}322 \\
(6.7)\end{array}$ & $\begin{array}{l}310 \\
(6.4)\end{array}$ & \\
\hline
\end{tabular}

P-value using ANOVA and Chi-square tests

Data are expressed as mean $\pm \mathrm{SD}$, or $\mathrm{n}(\%)$.

FPG, fasting plasma glucose; SD, standard deviation; BMI, Body mass index; BP, blood pressure; AST, aspartate aminotransferase; ALT, alanine aminotransferase; GGT, y-glutamyl transferase; CKD, chronic kidney disease 


\begin{tabular}{|c|c|c|c|c|c|c|c|c|c|c|}
\hline \multirow[b]{2}{*}{$\begin{array}{l}\text { Current } \\
\text { smoker }\end{array}$} & \multicolumn{5}{|c|}{ Midlife (Age < 65 years) } & \multicolumn{5}{|c|}{ Elderly (Age $\geq 65$ years) } \\
\hline & $\begin{array}{l}4593 \\
(16.4)\end{array}$ & $\begin{array}{l}5511 \\
(19.6)\end{array}$ & $\begin{array}{l}6118 \\
(21.8)\end{array}$ & $\begin{array}{l}6832 \\
(24.3)\end{array}$ & & $\begin{array}{l}486 \\
(10.3)\end{array}$ & $\begin{array}{l}543 \\
(11.0)\end{array}$ & $\begin{array}{l}579 \\
(12.0)\end{array}$ & $\begin{array}{l}665 \\
(13.8)\end{array}$ & \\
\hline $\begin{array}{l}\text { Alcohol } \\
\text { consumption } \\
(n, \%)\end{array}$ & $\begin{array}{l}11425 \\
(40.7)\end{array}$ & $\begin{array}{l}12781 \\
(45.5)\end{array}$ & $\begin{array}{l}13131 \\
(46.7)\end{array}$ & $\begin{array}{l}13470 \\
(47.9)\end{array}$ & $\begin{array}{l}< \\
0.001\end{array}$ & $\begin{array}{l}1137 \\
(24.2)\end{array}$ & $\begin{array}{l}1262 \\
(25.6)\end{array}$ & $\begin{array}{l}1319 \\
(27.4)\end{array}$ & $\begin{array}{l}1352 \\
(28.0)\end{array}$ & $\begin{array}{l}< \\
0.001\end{array}$ \\
\hline $\begin{array}{l}\text { Regular } \\
\text { exercise }(n, \\
\%)\end{array}$ & $\begin{array}{l}2702 \\
(9.6)\end{array}$ & $\begin{array}{l}2515 \\
(9.0)\end{array}$ & $\begin{array}{l}2469 \\
(8.8)\end{array}$ & $\begin{array}{l}2519 \\
(9.0)\end{array}$ & 0.003 & $\begin{array}{l}756 \\
(16.1)\end{array}$ & $\begin{array}{l}731 \\
(14.8)\end{array}$ & $\begin{array}{l}644 \\
(13.4)\end{array}$ & $\begin{array}{l}650 \\
(13.5)\end{array}$ & $\begin{array}{l}< \\
0.001\end{array}$ \\
\hline $\begin{array}{l}\text { Income } \\
(\text { lower } 10 \%) \\
(n, \%)\end{array}$ & $\begin{array}{l}1705 \\
(6.1)\end{array}$ & $\begin{array}{l}1801 \\
(6.4)\end{array}$ & $\begin{array}{l}1899 \\
(6.8)\end{array}$ & $\begin{array}{l}2271 \\
(8.1)\end{array}$ & $\begin{array}{l}< \\
0.001\end{array}$ & $\begin{array}{l}463 \\
(9.8)\end{array}$ & $\begin{array}{l}567 \\
(11.5)\end{array}$ & $\begin{array}{l}589 \\
(12.2)\end{array}$ & $\begin{array}{l}636 \\
(13.2)\end{array}$ & $\begin{array}{l}< \\
0.001\end{array}$ \\
\hline $\begin{array}{l}\text { Hypertension } \\
(\mathrm{n}, \%)\end{array}$ & $\begin{array}{l}13510 \\
(48.1)\end{array}$ & $\begin{array}{l}14641 \\
(52.1)\end{array}$ & $\begin{array}{l}15410 \\
(54.9)\end{array}$ & $\begin{array}{l}16533 \\
(58.9)\end{array}$ & $\begin{array}{l}< \\
0.001\end{array}$ & $\begin{array}{l}3529 \\
(75.0)\end{array}$ & $\begin{array}{l}3740 \\
(75.8)\end{array}$ & $\begin{array}{l}3703 \\
(76.9)\end{array}$ & $\begin{array}{l}3769 \\
(78.2)\end{array}$ & 0.002 \\
\hline $\begin{array}{l}\text { Dyslipidemia } \\
(\mathrm{n}, \%)\end{array}$ & $\begin{array}{l}8179 \\
(29.1)\end{array}$ & $\begin{array}{l}8535 \\
(30.4)\end{array}$ & $\begin{array}{l}8918 \\
(31.7)\end{array}$ & $\begin{array}{l}9442 \\
(33.6)\end{array}$ & $\begin{array}{l}< \\
0.001\end{array}$ & $\begin{array}{l}1779 \\
(37.8)\end{array}$ & $\begin{array}{l}1888 \\
(38.3)\end{array}$ & $\begin{array}{l}1878 \\
(39.0)\end{array}$ & $\begin{array}{l}1822 \\
(37.8)\end{array}$ & 0.597 \\
\hline $\begin{array}{l}\text { History of } \\
\text { stroke }(n, \%)\end{array}$ & $\begin{array}{l}111 \\
(0.4)\end{array}$ & $\begin{array}{l}120 \\
(0.4)\end{array}$ & $\begin{array}{l}130 \\
(0.5)\end{array}$ & $\begin{array}{l}134 \\
(0.5)\end{array}$ & 0.459 & $\begin{array}{l}85 \\
(1.8)\end{array}$ & $\begin{array}{l}91 \\
(1.8)\end{array}$ & $\begin{array}{l}85 \\
(1.8)\end{array}$ & $\begin{array}{l}91 \\
(1.9)\end{array}$ & 0.974 \\
\hline $\begin{array}{l}\text { History of } \\
\text { CKD }(n, \%)\end{array}$ & $\begin{array}{l}150 \\
(0.5)\end{array}$ & $\begin{array}{l}128 \\
(0.5)\end{array}$ & $\begin{array}{l}165 \\
(0.6)\end{array}$ & $\begin{array}{l}165 \\
(0.6)\end{array}$ & 0.109 & $\begin{array}{l}68 \\
(1.4)\end{array}$ & $\begin{array}{l}70 \\
(1.4)\end{array}$ & $\begin{array}{l}62 \\
(1.3)\end{array}$ & $\begin{array}{l}48 \\
(1.0)\end{array}$ & 0.185 \\
\hline $\begin{array}{l}\text { Use of anti- } \\
\text { hypertensive } \\
\text { agent }(n, \%)\end{array}$ & $\begin{array}{l}9427 \\
(33.6)\end{array}$ & $\begin{array}{l}9232 \\
(32.9)\end{array}$ & $\begin{array}{l}9460 \\
(33.7)\end{array}$ & $\begin{array}{l}10214 \\
(36.4)\end{array}$ & $\begin{array}{l}< \\
0.001\end{array}$ & $\begin{array}{l}3099 \\
(65.9)\end{array}$ & $\begin{array}{l}3260 \\
(66.1)\end{array}$ & $\begin{array}{l}3181 \\
(66.0)\end{array}$ & $\begin{array}{l}3244 \\
(67.3)\end{array}$ & 0.426 \\
\hline $\begin{array}{l}\text { Use of lipid- } \\
\text { lowering } \\
\text { agent }(n, \%)\end{array}$ & $\begin{array}{l}3193 \\
(11.4)\end{array}$ & $\begin{array}{l}3128 \\
(11.1)\end{array}$ & $\begin{array}{l}3254 \\
(11.6)\end{array}$ & $\begin{array}{l}3516 \\
(12.5)\end{array}$ & $\begin{array}{l}< \\
0.001\end{array}$ & $\begin{array}{l}976 \\
(20.7)\end{array}$ & $\begin{array}{l}1016 \\
(20.6)\end{array}$ & $\begin{array}{l}944 \\
(19.6)\end{array}$ & $\begin{array}{l}933 \\
(19.4)\end{array}$ & 0.222 \\
\hline \multicolumn{11}{|c|}{ P-value using ANOVA and Chi-square tests } \\
\hline \multicolumn{11}{|c|}{ Data are expressed as mean $\pm S D$, or $n(\%)$. } \\
\hline \multicolumn{11}{|c|}{$\begin{array}{l}\text { FPG, fasting plasma glucose; SD, standard deviation; BMI, Body mass index; BP, blood pressure; } A S T \text {, } \\
\text { aspartate aminotransferase; ALT, alanine aminotransferase; GGT, } \mathrm{Y} \text {-glutamyl transferase; CKD, chronic kidney } \\
\text { disease }\end{array}$} \\
\hline
\end{tabular}

\section{Implications of FPG variability with PD}

Table 2 presented the association between glucose variability, as determined by SD, CV, and VIM, with the risk of incident PD. In the midlife group, an incrementally higher risk of PD was observed in the higher variability quartile groups compared to the lowest quartile group in all models. This is so after adjusting for age, sex, BMI, hypertension, dyslipidemia, smoking status, alcohol consumption, regular exercise, income, history of stroke, history of CKD, and mean FPG (P for trend: FPG-SD 0.023, FPG-CV 0.046, and FPG-VIM 0.020). The adjusted HRs of incident PD in the highest quartile of the FPG variability group, as measured using SD, CV, and VIM, were 1.35 (95\% Cl, 1.07-1.70), 1.31 (95\% Cl, 1.04-1.65), and 1.33 (95\% Cl, 1.06-1.67), respectively, compared to the lowest quartile group. However, in the elderly group, there was no significant difference in incident PD according to the quartiles of FPG variability before and after adjusting for multi-variables and mean FPG. 
Table 2

Hazard ratios (HR) and 95\% confidence intervals (Cls) of Parkinson's disease by quartiles of FPG variability (SD, $\mathrm{CV}$, and VIM)

Midlife (Age $<65$ years)

$\begin{array}{llll}\text { Events } & \text { Follow- } & \text { Incidence } & \text { Adjusted } \\ \text { (n) } & \text { up } & \text { rate }(\text { per } & \text { HR } \\ & \text { duration } & 1000 & (95 \% \mathrm{Cl}) \\ & \text { (person } & \text { person- } & \\ & \text { years) } & \text { years) } & \end{array}$

\section{Elderly (Age $\geq 65$ years)}

\begin{tabular}{|c|c|c|c|}
\hline $\begin{array}{l}\text { Events } \\
\text { (n) }\end{array}$ & $\begin{array}{l}\text { Follow-up } \\
\text { duration } \\
\text { (person } \\
\text { years) }\end{array}$ & $\begin{array}{l}\text { Incidence } \\
\text { rate (per } \\
1000 \\
\text { person- } \\
\text { years) }\end{array}$ & $\begin{array}{l}\text { Adjusted } \\
\text { HR } \\
(95 \% \mathrm{Cl})\end{array}$ \\
\hline
\end{tabular}

FPG-SD

$\begin{array}{lllllllll}\text { Q1 } & 130 & 234,031 & 0.56 & 1 \text { (ref) } & 204 & 37,305 & 5.47 & 1 \text { (ref) } \\ \text { Q2 } & 130 & 233,586 & 0.56 & \begin{array}{l}1.08 \\ (0.84-\end{array} & 146 & 39,377 & 3.71 & \begin{array}{l}0.69(0.56- \\ 1.37)\end{array} \\ & & & & & & 0.85) \\ \text { Q3 } & 120 & 233,469 & 0.51 & \begin{array}{l}0.98 \\ (0.77-\end{array} & 175 & 38,235 & 4.58 & 0.85(0.70- \\ & & & & & & 1.04) \\ \text { Q4 } & 174 & 232,812 & 0.75 & \begin{array}{l}1.35 \\ (1.07-\end{array} & 184 & 37,676 & 4.88 & 0.90(0.74- \\ & & & & & & & & 1.10)\end{array}$

P for

0.023

0.647

trend

FPG-CV

\begin{tabular}{|c|c|c|c|c|c|c|c|c|}
\hline Q1 & 129 & 233,983 & 0.55 & 1 (ref) & 207 & 38,316 & 5.4 & 1 (ref) \\
\hline Q2 & 135 & 233,548 & 0.58 & $\begin{array}{l}1.13 \\
(0.89- \\
1.44)\end{array}$ & 144 & 38,345 & 3.76 & $\begin{array}{l}0.71(0.58- \\
0.88)\end{array}$ \\
\hline Q3 & 124 & 233,461 & 0.53 & $\begin{array}{l}1.03 \\
(0.80- \\
1.32)\end{array}$ & 179 & 38,306 & 4.67 & $\begin{array}{l}0.88(0.72- \\
1.08)\end{array}$ \\
\hline Q4 & 166 & 232,906 & 0.71 & $\begin{array}{l}1.31 \\
(1.04- \\
1.65)\end{array}$ & 179 & 37,627 & 4.76 & $\begin{array}{l}0.89(0.73- \\
1.09)\end{array}$ \\
\hline
\end{tabular}

P for

0.046

0.546

trend

FPG-VIM

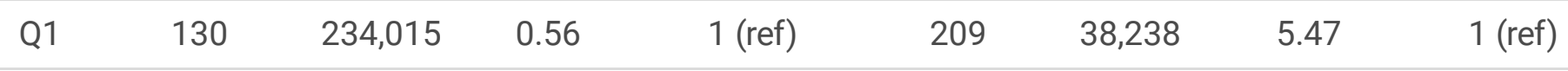

Adjusted for age, sex, body mass index, hypertension, dyslipidemia, smoking status, alcohol consumption, regular exercise, income, history of stroke, history of CKD, and mean FPG

FPG, fasting plasma glucose; SD, standard deviation; CV, coefficient of variation; VIM, variability independent of the mean 


\begin{tabular}{|c|c|c|c|c|c|c|c|c|}
\hline \multirow[b]{2}{*}{ Q2 } & \multicolumn{4}{|c|}{ Midlife (Age $<65$ years) } & \multicolumn{4}{|c|}{ Elderly (Age $\geq 65$ years) } \\
\hline & 127 & 233,643 & 0.54 & $\begin{array}{l}1.05 \\
(0.82- \\
1.34)\end{array}$ & 140 & 38,439 & 3.64 & $\begin{array}{l}0.68(0.55- \\
0.85)\end{array}$ \\
\hline Q3 & 126 & 233,410 & 0.54 & $\begin{array}{l}1.03 \\
(0.81- \\
1.32)\end{array}$ & 176 & 38,237 & 4.6 & $\begin{array}{l}0.86(0.70- \\
1.05)\end{array}$ \\
\hline Q4 & 171 & 232,829 & 0.73 & $\begin{array}{l}1.33 \\
(1.06- \\
1.67)\end{array}$ & 184 & 37,680 & 4.88 & $\begin{array}{l}0.90(0.74- \\
1.10)\end{array}$ \\
\hline $\begin{array}{l}\text { P for } \\
\text { trend }\end{array}$ & & & & 0.020 & & & & 0.654 \\
\hline \multicolumn{9}{|c|}{$\begin{array}{l}\text { Adjusted for age, sex, body mass index, hypertension, dyslipidemia, smoking status, alcohol consumption, } \\
\text { regular exercise, income, history of stroke, history of CKD, and mean FPG }\end{array}$} \\
\hline \multicolumn{9}{|c|}{$\begin{array}{l}\text { FPG, fasting plasma glucose; SD, standard deviation; CV, coefficient of variation; VIM, variability independent } \\
\text { of the mean }\end{array}$} \\
\hline
\end{tabular}

Figure 1 shows the Kaplan-Meier curves of the probability of incident PD for 8 years according to the levels of FPG variability, measured as FPG-SD, FPG-CV, and FPG-VIM, in the midlife group. Higher levels of FPG variability were associated with an increased risk of developing PD progressively (log rank P: FPG-SD 0.005, FPG-CV 0.048, and FPG-VIM 0.015).

Sensitivity analyses excluding participants who were diagnosed dementia (Table 3 ) or stroke (Table 4), also showed that increased risk for incident PD in the highest quartile group are maintained compared to the lowest quartile group in the midlife group. 
Table 3

Hazard ratios (HR) and 95\% confidence intervals (Cls) of Parkinson's disease by quartiles of FPG variability in the midlife group without dementia

\begin{tabular}{|c|c|c|c|c|c|c|c|c|c|}
\hline & \multirow{2}{*}{$\begin{array}{l}\text { Number of } \\
\text { participants }\end{array}$} & \multirow{2}{*}{$\begin{array}{l}\text { Number } \\
\text { of } \\
\text { events }\end{array}$} & \multirow{2}{*}{$\begin{array}{l}\text { Follow- } \\
\text { up } \\
\text { duration } \\
\text { (person } \\
\text { years) }\end{array}$} & \multirow{2}{*}{$\begin{array}{l}\text { Incidence } \\
\text { rate (per } \\
1000 \\
\text { person- } \\
\text { years) }\end{array}$} & \multicolumn{5}{|c|}{ Hazard Ratio (95\% Cl) } \\
\hline & & & & & Unadjusted & $\begin{array}{l}\text { Model } \\
1 *\end{array}$ & $\begin{array}{l}\text { Model } \\
2+\end{array}$ & $\begin{array}{l}\text { Model } \\
3 \neq\end{array}$ & $\begin{array}{l}\text { Model } \\
4 \S\end{array}$ \\
\hline \multicolumn{10}{|c|}{ FPG-SD } \\
\hline Q1 & 28074 & 129 & 233,924 & 0.55 & 1 (ref) & 1 (ref) & 1 (ref) & 1 (ref) & 1 (ref) \\
\hline Q2 & 28086 & 130 & 233,674 & 0.56 & $\begin{array}{l}1.01 \\
(0.79- \\
1.29)\end{array}$ & $\begin{array}{l}1.09 \\
(0.86- \\
1.40)\end{array}$ & $\begin{array}{l}1.09 \\
(0.85- \\
1.39)\end{array}$ & $\begin{array}{l}1.08 \\
(0.85- \\
1.38)\end{array}$ & $\begin{array}{l}1.08 \\
(0.85- \\
1.38)\end{array}$ \\
\hline Q3 & 28064 & 120 & 233,233 & 0.51 & $\begin{array}{l}0.93 \\
(0.73- \\
1.20)\end{array}$ & $\begin{array}{l}1.01 \\
(0.78- \\
1.29)\end{array}$ & $\begin{array}{l}0.99 \\
(0.77- \\
1.27)\end{array}$ & $\begin{array}{l}0.99 \\
(0.77- \\
1.27)\end{array}$ & $\begin{array}{l}0.99 \\
(0.77- \\
1.27)\end{array}$ \\
\hline Q4 & 28082 & 171 & 232,747 & 0.73 & $\begin{array}{l}1.33 \\
(1.06- \\
1.68)\end{array}$ & $\begin{array}{l}1.36 \\
(1.08- \\
1.72)\end{array}$ & $\begin{array}{l}1.34 \\
(1.06- \\
1.68)\end{array}$ & $\begin{array}{l}1.34 \\
(1.06- \\
1.68)\end{array}$ & $\begin{array}{l}1.34 \\
(1.06- \\
1.69)\end{array}$ \\
\hline$P f c$ & trend & & & & 0.024 & 0.017 & 0.027 & 0.027 & 0.028 \\
\hline \multicolumn{10}{|c|}{ FPG-CV } \\
\hline Q1 & 28076 & 128 & 233,902 & 0.55 & 1 (ref) & 1 (ref) & 1 (ref) & 1 (ref) & 1 (ref) \\
\hline Q2 & 28076 & 135 & 233,459 & 0.58 & $\begin{array}{l}1.06 \\
(0.83- \\
1.35)\end{array}$ & $\begin{array}{l}1.15 \\
(0.90- \\
1.46)\end{array}$ & $\begin{array}{l}1.14 \\
(0.89- \\
1.45)\end{array}$ & $\begin{array}{l}1.14 \\
(0.89- \\
1.45)\end{array}$ & $\begin{array}{l}1.14 \\
(0.89- \\
1.45)\end{array}$ \\
\hline Q3 & 28078 & 123 & 233,389 & 0.53 & $\begin{array}{l}0.96 \\
(0.75- \\
1.23)\end{array}$ & $\begin{array}{l}1.04 \\
(0.81- \\
1.34)\end{array}$ & $\begin{array}{l}1.03 \\
(0.80- \\
1.32)\end{array}$ & $\begin{array}{l}1.03 \\
(0.80- \\
1.32)\end{array}$ & $\begin{array}{l}1.03 \\
(0.80- \\
1.32)\end{array}$ \\
\hline Q4 & 28076 & 164 & 232,829 & 0.70 & $\begin{array}{l}1.29 \\
(1.02- \\
1.62)\end{array}$ & $\begin{array}{l}1.33 \\
(1.05- \\
1.68)\end{array}$ & $\begin{array}{l}1.30 \\
(1.03- \\
1.65)\end{array}$ & $\begin{array}{l}1.30 \\
(1.03- \\
1.65)\end{array}$ & $\begin{array}{l}1.31 \\
(1.04- \\
1.65)\end{array}$ \\
\hline$P f c$ & trend & & & & 0.061 & 0.036 & 0.055 & 0.056 & 0.054 \\
\hline \multicolumn{10}{|c|}{ FPG-VIM } \\
\hline Q1 & 28076 & 129 & 233,926 & 0.55 & 1 (ref) & 1 (ref) & 1 (ref) & 1 (ref) & 1 (ref) \\
\hline
\end{tabular}

*Model 1: Adjusted for age and sex. †Model 2: Adjusted for age, sex, body mass index, hypertension, dyslipidemia, smoking, alcohol consumption, regular exercise, and income. ¥Model 3: Adjusted for age, sex, body mass index, hypertension, dyslipidemia, smoking, alcohol consumption, regular exercise, income, history of stroke, and history of CKD. §Model 4: Adjusted for age, sex, body mass index, hypertension, dyslipidemia, smoking, alcohol consumption, regular exercise, income, history of stroke, history of CKD, and mean FPG

FPG, fasting plasma glucose; CKD, chronic kidney disease; SD, standard deviation; CV, coefficient of variation; VIM, variability independent of the mean. 


\begin{tabular}{|c|c|c|c|c|c|c|c|c|c|}
\hline & \multirow{2}{*}{$\begin{array}{l}\text { Number of } \\
\text { participants }\end{array}$} & \multirow{2}{*}{$\begin{array}{l}\text { Number } \\
\text { of } \\
\text { events }\end{array}$} & \multirow{2}{*}{$\begin{array}{l}\text { Follow- } \\
\text { up } \\
\text { duration } \\
\text { (person } \\
\text { years) }\end{array}$} & \multirow{2}{*}{$\begin{array}{l}\text { Incidence } \\
\text { rate (per } \\
1000 \\
\text { person- } \\
\text { years) }\end{array}$} & \multicolumn{5}{|c|}{ Hazard Ratio $(95 \% \mathrm{Cl})$} \\
\hline & & & & & Unadjusted & $\begin{array}{l}\text { Model } \\
1^{*}\end{array}$ & $\begin{array}{l}\text { Model } \\
2+\end{array}$ & $\begin{array}{l}\text { Model } \\
3 \neq\end{array}$ & $\begin{array}{l}\text { Model } \\
4 \S\end{array}$ \\
\hline Q2 & 28077 & 127 & 233,569 & 0.54 & $\begin{array}{l}0.99 \\
(0.77- \\
1.26)\end{array}$ & $\begin{array}{l}1.07 \\
(0.84- \\
1.37)\end{array}$ & $\begin{array}{l}1.06 \\
(0.83- \\
1.36)\end{array}$ & $\begin{array}{l}1.06 \\
(0.83- \\
1.35)\end{array}$ & $\begin{array}{l}1.06 \\
(0.83- \\
1.35)\end{array}$ \\
\hline Q3 & 28077 & 126 & 233,321 & 0.54 & $\begin{array}{l}0.98 \\
(0.77- \\
1.25)\end{array}$ & $\begin{array}{l}1.06 \\
(0.83- \\
1.35)\end{array}$ & $\begin{array}{l}1.04 \\
(0.82- \\
1.34)\end{array}$ & $\begin{array}{l}1.04 \\
(0.82- \\
1.34)\end{array}$ & $\begin{array}{l}1.04 \\
(0.82- \\
1.34)\end{array}$ \\
\hline Q4 & 28076 & 168 & 232,763 & 0.72 & $\begin{array}{l}1.31 \\
(1.04- \\
1.65)\end{array}$ & $\begin{array}{l}1.34 \\
(1.07- \\
1.69)\end{array}$ & $\begin{array}{l}1.32 \\
(1.05- \\
1.66)\end{array}$ & $\begin{array}{l}1.32 \\
(1.05- \\
1.66)\end{array}$ & $\begin{array}{l}1.32 \\
(1.04- \\
1.66)\end{array}$ \\
\hline \multicolumn{5}{|c|}{$P$ for trend } & 0.024 & 0.016 & 0.025 & 0.025 & 0.025 \\
\hline \multicolumn{10}{|c|}{$\begin{array}{l}\text { *Model 1: Adjusted for age and sex. †Model 2: Adjusted for age, sex, body mass index, hypertension, } \\
\text { dyslipidemia, smoking, alcohol consumption, regular exercise, and income. ¥Model 3: Adjusted for age, sex, } \\
\text { body mass index, hypertension, dyslipidemia, smoking, alcohol consumption, regular exercise, income, history } \\
\text { of stroke, and history of CKD. SModel 4: Adjusted for age, sex, body mass index, hypertension, dyslipidemia, } \\
\text { smoking, alcohol consumption, regular exercise, income, history of stroke, history of CKD, and mean FPG }\end{array}$} \\
\hline \multicolumn{10}{|c|}{$\begin{array}{l}\text { FPG, fasting plasma glucose; CKD, chronic kidney disease; SD, standard deviation; CV, coefficient of variation; } \\
\text { VIM, variability independent of the mean. }\end{array}$} \\
\hline
\end{tabular}


Table 4

Hazard ratios (HR) and 95\% confidence intervals (Cls) of Parkinson's disease by quartiles of FPG variability in the midlife group without stroke

\begin{tabular}{|c|c|c|c|c|c|c|c|c|c|}
\hline & \multirow{2}{*}{$\begin{array}{l}\text { Number of } \\
\text { participants }\end{array}$} & \multirow{2}{*}{$\begin{array}{l}\text { Number } \\
\text { of } \\
\text { events }\end{array}$} & \multirow{2}{*}{$\begin{array}{l}\text { Follow- } \\
\text { up } \\
\text { duration } \\
\text { (person } \\
\text { years) }\end{array}$} & \multirow{2}{*}{$\begin{array}{l}\text { Incidence } \\
\text { rate (per } \\
1000 \\
\text { person- } \\
\text { years) }\end{array}$} & \multicolumn{5}{|c|}{ Hazard Ratio $(95 \% \mathrm{Cl})$} \\
\hline & & & & & Unadjusted & $\begin{array}{l}\text { Model } \\
1^{*}\end{array}$ & $\begin{array}{l}\text { Model } \\
2+\end{array}$ & $\begin{array}{l}\text { Model } \\
3 \neq\end{array}$ & $\begin{array}{l}\text { Model } \\
4 \S\end{array}$ \\
\hline \multicolumn{10}{|c|}{ FPG-SD } \\
\hline Q1 & 27962 & 129 & 232,993 & 0.55 & 1 (ref) & 1 (ref) & 1 (ref) & 1 (ref) & 1 (ref) \\
\hline Q2 & 27970 & 129 & 232,693 & 0.55 & $\begin{array}{l}1.00 \\
(0.78- \\
1.28)\end{array}$ & $\begin{array}{l}1.09 \\
(0.85- \\
1.39)\end{array}$ & $\begin{array}{l}1.08 \\
(0.84- \\
1.37)\end{array}$ & $\begin{array}{l}1.08 \\
(0.84- \\
1.37)\end{array}$ & $\begin{array}{l}1.08 \\
(0.84- \\
1.37)\end{array}$ \\
\hline Q3 & 27962 & 117 & 232,383 & 0.50 & $\begin{array}{l}0.91 \\
(0.71- \\
1.17)\end{array}$ & $\begin{array}{l}0.98 \\
(0.76- \\
1.26)\end{array}$ & $\begin{array}{l}0.97 \\
(0.75- \\
1.24)\end{array}$ & $\begin{array}{l}0.97 \\
(0.75- \\
1.24)\end{array}$ & $\begin{array}{l}0.97 \\
(0.75- \\
1.24)\end{array}$ \\
\hline Q4 & 27958 & 171 & 231,743 & 0.74 & $\begin{array}{l}1.33 \\
(1.06- \\
1.68)\end{array}$ & $\begin{array}{l}1.36 \\
(1.08- \\
1.71)\end{array}$ & $\begin{array}{l}1.34 \\
(1.06- \\
1.68)\end{array}$ & $\begin{array}{l}1.34 \\
(1.06- \\
1.68)\end{array}$ & $\begin{array}{l}1.34 \\
(1.06- \\
1.69)\end{array}$ \\
\hline$P$ fo & trend & & & & 0.026 & 0.019 & 0.029 & 0.029 & 0.030 \\
\hline \multicolumn{10}{|c|}{ FPG-CV } \\
\hline Q1 & 27963 & 127 & 232,970 & 0.55 & 1 (ref) & 1 (ref) & 1 (ref) & 1 (ref) & 1 (ref) \\
\hline Q2 & 27963 & 134 & 232,503 & 0.58 & $\begin{array}{l}1.06 \\
(0.83- \\
1.35)\end{array}$ & $\begin{array}{l}1.15 \\
(0.90- \\
1.46)\end{array}$ & $\begin{array}{l}1.14 \\
(0.89- \\
1.45)\end{array}$ & $\begin{array}{l}1.14 \\
(0.89- \\
1.45)\end{array}$ & $\begin{array}{l}1.14 \\
(0.89- \\
1.45)\end{array}$ \\
\hline Q3 & 27963 & 122 & 232,422 & 0.52 & $\begin{array}{l}0.96 \\
(0.75- \\
1.24)\end{array}$ & $\begin{array}{l}1.04 \\
(0.81- \\
1.34)\end{array}$ & $\begin{array}{l}1.03 \\
(0.80- \\
1.32)\end{array}$ & $\begin{array}{l}1.03 \\
(0.80- \\
1.32)\end{array}$ & $\begin{array}{l}1.03 \\
(0.80- \\
1.32)\end{array}$ \\
\hline Q4 & 27963 & 163 & 231,917 & 0.70 & $\begin{array}{l}1.29 \\
(1.02- \\
1.63)\end{array}$ & $\begin{array}{l}1.33 \\
(1.05- \\
1.68)\end{array}$ & $\begin{array}{l}1.31 \\
(1.03- \\
1.65)\end{array}$ & $\begin{array}{l}1.31 \\
(1.03- \\
1.65)\end{array}$ & $\begin{array}{l}1.31 \\
(1.04- \\
1.65)\end{array}$ \\
\hline$P$ fo & trend & & & & 0.060 & 0.037 & 0.055 & 0.055 & 0.052 \\
\hline \multicolumn{10}{|c|}{ FPG-VIM } \\
\hline Q1 & 27963 & 129 & 232,984 & 0.55 & 1 (ref) & 1 (ref) & 1 (ref) & 1 (ref) & 1 (ref) \\
\hline
\end{tabular}

*Model 1: Adjusted for age and sex. †Model 2: Adjusted for age, sex, body mass index, hypertension, dyslipidemia, smoking, alcohol consumption, regular exercise, and income. ¥Model 3: Adjusted for age, sex, body mass index, hypertension, dyslipidemia, smoking, alcohol consumption, regular exercise, income, and history of CKD. §Model 4: Adjusted for age, sex, body mass index, hypertension, dyslipidemia, smoking, alcohol consumption, regular exercise, income, history of CKD, and mean FPG

FPG, fasting plasma glucose; CKD, chronic kidney disease; SD, standard deviation; CV, coefficient of variation; $\mathrm{VIM}$, variability independent of the mean. 


\begin{tabular}{|c|c|c|c|c|c|c|c|c|c|}
\hline & \multirow{2}{*}{$\begin{array}{l}\text { Number of } \\
\text { participants }\end{array}$} & \multirow{2}{*}{$\begin{array}{l}\text { Number } \\
\text { of } \\
\text { events }\end{array}$} & \multirow{2}{*}{$\begin{array}{l}\text { Follow- } \\
\text { up } \\
\text { duration } \\
\text { (person } \\
\text { years) }\end{array}$} & \multirow{2}{*}{$\begin{array}{l}\text { Incidence } \\
\text { rate (per } \\
1000 \\
\text { person- } \\
\text { years) }\end{array}$} & \multicolumn{5}{|c|}{ Hazard Ratio $(95 \% \mathrm{Cl})$} \\
\hline & & & & & Unadjusted & $\begin{array}{l}\text { Model } \\
1 *\end{array}$ & $\begin{array}{l}\text { Model } \\
2 \dagger\end{array}$ & $\begin{array}{l}\text { Model } \\
3 \neq\end{array}$ & $\begin{array}{l}\text { Model } \\
4 \S\end{array}$ \\
\hline Q2 & 27963 & 126 & 232,610 & 0.54 & $\begin{array}{l}0.98 \\
(0.77- \\
1.25)\end{array}$ & $\begin{array}{l}1.06 \\
(0.83- \\
1.36)\end{array}$ & $\begin{array}{l}1.05 \\
(0.82- \\
1.35)\end{array}$ & $\begin{array}{l}1.05 \\
(0.82- \\
1.34)\end{array}$ & $\begin{array}{l}1.05 \\
(0.82- \\
1.35)\end{array}$ \\
\hline Q3 & 27964 & 123 & 232,384 & 0.53 & $\begin{array}{l}0.96 \\
(0.75- \\
1.22)\end{array}$ & $\begin{array}{l}1.03 \\
(0.81- \\
1.32)\end{array}$ & $\begin{array}{l}1.02 \\
(0.79- \\
1.30)\end{array}$ & $\begin{array}{l}1.02 \\
(0.79- \\
1.31)\end{array}$ & $\begin{array}{l}1.02 \\
(0.79- \\
1.31)\end{array}$ \\
\hline Q4 & 27962 & 168 & 231,835 & 0.72 & $\begin{array}{l}1.31 \\
(1.04- \\
1.65)\end{array}$ & $\begin{array}{l}1.34 \\
(1.07- \\
1.69)\end{array}$ & $\begin{array}{l}1.32 \\
(1.04- \\
1.66)\end{array}$ & $\begin{array}{l}1.32 \\
(1.05- \\
1.66)\end{array}$ & $\begin{array}{l}1.32 \\
(1.04- \\
1.66)\end{array}$ \\
\hline \multicolumn{5}{|c|}{$P$ for trend } & 0.026 & 0.018 & 0.027 & 0.027 & 0.027 \\
\hline \multicolumn{10}{|c|}{ 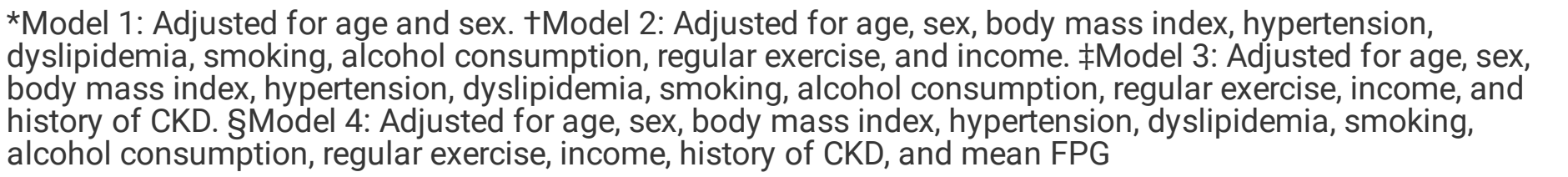 } \\
\hline \multicolumn{10}{|c|}{$\begin{array}{l}\text { FPG, fasting plasma glucose; CKD, chronic kidney disease; SD, standard deviation; CV, coefficient of variation; } \\
\text { VIM, variability independent of the mean. }\end{array}$} \\
\hline
\end{tabular}

Within the midlife group, a 1 SD-increment of PFG variability was associated with an increased risk of PD in all models (Table 5). Moreover, these associations persisted after stepwise adjustment for exposure to PD risk factors in all models and after adjusting for diverse confounding factors and mean FPG levels. We observed a significant association with a 1-SD unit increment in FPG variability in model 4 , including adjustment for mean FPG level: FPG-SD: 1.09 (95\% Cl, 1.01-1.18; P = 0.036), FPG-CV: 1.09 (95\% Cl, 1.00-1.18; P = 0.040), and FPG-VIM: $1.09(95 \% \mathrm{Cl}, 1.00-1.18 ; \mathrm{P}=0.038)$. 
Table 5

Risk of Parkinson's disease according to 1-SD change of FPG variability (SD, CV, and VIM) in the midlife group

\begin{tabular}{|c|c|c|c|c|c|c|c|c|c|c|}
\hline & \multicolumn{2}{|c|}{ Unadjusted } & \multicolumn{2}{|c|}{ Model 1} & \multicolumn{2}{|c|}{ Model 2} & \multicolumn{2}{|c|}{ Model 3} & \multicolumn{2}{|c|}{ Model 4} \\
\hline & $\begin{array}{l}\mathrm{HR} \\
(95 \% \mathrm{Cl})\end{array}$ & $\begin{array}{l}\mathrm{P}- \\
\text { value }\end{array}$ & $\begin{array}{l}\text { HR } \\
(95 \% \\
\mathrm{Cl})\end{array}$ & $\begin{array}{l}\mathrm{P}- \\
\text { value }\end{array}$ & $\begin{array}{l}\mathrm{HR} \\
(95 \% \\
\mathrm{Cl})\end{array}$ & $\begin{array}{l}\mathrm{P}- \\
\text { value }\end{array}$ & $\begin{array}{l}\mathrm{HR} \\
(95 \% \\
\mathrm{Cl})\end{array}$ & $\begin{array}{l}\text { P- } \\
\text { value }\end{array}$ & $\begin{array}{l}\mathrm{HR} \\
(95 \% \\
\mathrm{Cl})\end{array}$ & $\begin{array}{l}\text { P- } \\
\text { value }\end{array}$ \\
\hline $\begin{array}{l}\text { FPG- } \\
\text { SD }\end{array}$ & $\begin{array}{l}1.11 \\
(1.02- \\
1.20)\end{array}$ & 0.015 & $\begin{array}{l}1.10 \\
(1.01- \\
1.19)\end{array}$ & 0.021 & $\begin{array}{l}1.09 \\
(1.01- \\
1.18)\end{array}$ & 0.033 & $\begin{array}{l}1.09 \\
(1.01- \\
1.18)\end{array}$ & 0.034 & $\begin{array}{l}1.09 \\
(1.01- \\
1.18)\end{array}$ & 0.036 \\
\hline $\begin{array}{l}\text { FPG- } \\
\text { CV }\end{array}$ & $\begin{array}{l}1.09 \\
(1.01- \\
1.18)\end{array}$ & 0.030 & $\begin{array}{l}1.09 \\
(1.01- \\
1.18)\end{array}$ & 0.027 & $\begin{array}{l}1.09 \\
(1.00- \\
1.17)\end{array}$ & 0.042 & $\begin{array}{l}1.08 \\
(1.00- \\
1.17)\end{array}$ & 0.043 & $\begin{array}{l}1.09 \\
(1.00- \\
1.18)\end{array}$ & 0.040 \\
\hline $\begin{array}{l}\text { FPG- } \\
\text { VIM }\end{array}$ & $\begin{array}{l}1.10 \\
(1.01- \\
1.19)\end{array}$ & 0.021 & $\begin{array}{l}1.09 \\
(1.01- \\
1.18)\end{array}$ & 0.023 & $\begin{array}{l}1.09 \\
(1.01- \\
1.18)\end{array}$ & 0.036 & $\begin{array}{l}1.09 \\
(1.01- \\
1.18)\end{array}$ & 0.037 & $\begin{array}{l}1.09 \\
(1.00- \\
1.18)\end{array}$ & 0.038 \\
\hline
\end{tabular}

Model 1: Adjusted for age and sex

Model 2: Adjusted for age, sex, body mass index, hypertension, dyslipidemia, smoking, alcohol intake, regular exercise, and income

Model 3: Adjusted for age, sex, body mass index, hypertension, dyslipidemia, smoking, alcohol intake, regular exercise, income, history of stroke, and history of CKD

Model 4: Adjusted for age, sex, body mass index, hypertension, dyslipidemia, smoking, alcohol intake, regular exercise, income, history of stroke, history of CKD, and mean FPG

FPG, fasting plasma glucose; SD, standard deviation; CV, coefficient of variation; VIM, variability independent of the mean; CKD, chronic kidney disease

\section{Discussion}

This nationwide population-based study first demonstrated that long-term visit-to-visit FPG variability in the midlife group is a meaningful predictor of PD occurrence during the follow-up period of 8.4 years. Additionally, we demonstrated that there is an independent association of greater FPG variability with higher risk of incident PD, even after adjusting for confounding risk factors including mean FPG level, indicating that this result may be robust. Moreover, with a 1-SD unit increment in glycemic variability, there is a $9 \%$ higher risk for incident PD after adjusting for established PD risk factors in the midlife group. Meanwhile, no relationship was found between glycemic variability and a future risk of PD in the elderly group.

Several previous studies focused on diabetic patients have demonstrated the link between intraindividual glycemic variability and the risk of diabetes-related adverse outcomes. In the intensive glucose treatment group of the Action in Diabetes and Vascular Disease: Preterax and Diamicron MR Controlled Evaluation (ADVANCE) trial, increased Hemoglobin A1C (HbA1c)-SD and FPG-SD were related to a high risk of microvascular and macrovascular events and mortality [14]. Moreover, it is showed that not only severe hypoglycemic events [22] but also peak glucose levels [23] are strongly related to the risk of dementia, the most common neurodegenerative disease, in subjects with diabetes. In the Taiwan Diabetes Study cohort $(n=16,709)$, an increased glycemic variability, determined by FPG-CV and $\mathrm{HbA} 1 \mathrm{c}-\mathrm{CV}$, were independently related to an elevated 
risk of developing Alzheimer's disease (AD) during a median follow-up of period of 8.9 years [24]. Additionally, in the same cohort, greater glycemic variability was associated with higher risk of ischemic stroke [25] and endstage renal disease [13] In a meta-analysis study, Gorst et al. reported that a high long-term HbA1c variability was significantly associated with micro- and macrovascular complications and increased incident mortality in patients with type 1 and type 2 diabetes [12].

However, there have been limited studies evaluating the effect of long-term glycemic variability in subjects without diabetes. In a study of the Chinese population (including diabatic patients, 2.5\%), visit-to-visit FPG variability was positively associated with the risk of CVD and all-cause mortality, even after adjusting for confounding factors such as mean FPG level [15]. Recently, Bancks et al. reported an association between longterm FPG variability and decreased cognitive function, after adjusting for mean FPG level in the subjects without diabetes, among the Coronary Artery Risk Development in Young Adults (CARDIA) Study cohort [26]. In the present study, we found that long-term FPG variability is independently associated with the risk of developing PD in the middle-aged population without diabetes. In particular, the subjects with highest quartile of FPG variability consistently showed an increased the risk of developing PD by over 30\% compared to those with the lowest quartile in all three different indicators such as FPG-SD, FPG-CV, and FPG-VIM. Moreover, multivariable linear regression showed a significantly increased risk of future PD based on a 1-SD change of glycemic variability, regardless of the calculating method of variability (e.g, SD, CV, and VIM), in the midlife group. Even with a stepwise adjustment for clinical and behavior risk factors, the degree of increased risk for PD did not change meaningfully. Therefore, long-term glycemic variability may act as a predictor for the future risk of PD as we showed that this relationship persisted despite adjusting for various confounding factors including mean FPG level.

In this study, the association between glycemic variability and the future risk of PD was only evident in the midlife group. Although we could not provide the exact reason for this age-dependent effect, growing evidence has shown that glucose metabolic disorders have a greater effect on neurodegenerative diseases in midlife populations than elderly populations. In Taiwan's population-based cohort, stratified by sex and age, diabetes was significantly linked to an elevated risk for incident PD; particularly, the association was stronger in the midlife population (<60 years) [4]. Similar to Taiwan's study, a Danish study found the risk of developing PD was significantly higher among subjects with diabetes if early-onset ( $<60$ years) rather than late-onset PD ( $\geq$ 60 years) [6]. Likewise, Arvanitakis et al. demonstrated that the association between diabetes and parkinsonian signs was more pronounced in the midlife group than in the elderly group [27]. Furthermore, in 3,307 black and Caucasian subjects without diabetes, a higher FPG variability during young adulthood was related to poorer cognitive functioning in midlife, independent of FPG levels [26].

There are some potential mechanisms that may explain the effect of glycemic variability on the development PD. Reactive oxygen species (ROS) production coupled with insufficient antioxidant defense can lead to oxidative stress; this causes various pathophysiological conditions such as aging, type 2 diabetes, and neurodegenerative diseases (e.g., PD and AD) [28]. DNA damage originating from ROS is a conspicuous feature of PD [29]. Previously, it is suggested the vicious cycle among glycemic variability, oxidative stress, increased proinflammatory cytokine, and insulin resistance. Some studies showed that increased glycemic variability caused more extreme oxidative stress than chronic hyperglycemia in the subjects with or without type 2 diabetes [10,11]. Abrupt hyperglycemia stimulates inflammatory cytokines including interleukin- 6 and tumor necrosis factor-alpha via oxidative stress [30]. Concurrently, hyperglycemia leads to insulin over-secretion by compensatory

Page $16 / 23$ 
mechanisms, which may lead to hypoglycemic events, increasing peripheral or central insulin resistance [31]. Further, this can cause vascular damage through the aggravation of hypertension, dyslipidemia and the stimulation of the proliferation and migration of arterial smooth muscle cells [32]. Moreover, accumulating evidence has revealed that the insulin signaling pathway may be central to the pathogenesis of PD [33]. It is known that insulin can pass through the blood-brain-barrier; insulin receptors exist in the substantia nigra and basal ganglia [34]. Much less insulin receptor mRNA expression was found in the substantia nigra from brain tissue affected by PD compared with tissue from normal controls [35]. Insulin exhibits neuroprotective effects by modulating neuronal growth and survival, maintenance of synapses, and dopaminergic transmission. Conversely, defective insulin signaling pathways can lead to the downregulation of the AKT pathway, mitochondrial dysfunction, oxidative stress, inflammation, decreased expression of peroxisome proliferatoractivated receptor-gamma coactivator $1 a$, and the increase of a-synuclein in the brain [33,36]. Additionally, both peripheral and neuronal insulin resistance, induced by metabolic stress (e.g., glucose fluctuation and inflammation), contribute to reduced dopaminergic signaling and increased nigrostriatal neurodegeneration, which results in the progression of PD $[33,34,37,38]$. Several antidiabetic medications have demonstrated beneficial effects on the outcomes of PD in animal models and in early-phase clinical trials. In in vitro and in vivo animal models of PD, both dipeptidyl peptidase-4 (DPP-4) inhibitors and glucagon-like peptide-1 receptor agonists showed neuroprotective effects [39]. Interestingly, DPP-4 inhibitors were reported to be more effective in reducing glycemic variability compared to sulfonylurea in patients with type 2 diabetes [40]. In a nationwide casecontrol study, Svenningsson et al. found a significantly decreased incidence of PD in patients taking DPP-4 inhibitors [41].

This study has several limitations to be considered. First, although we attempted to adjust for multiple confounding variables that influence the development of PD, using multivariate analyses, the possibility of unmeasured or residual confounding factors remain. Second, methods of measuring blood glucose levels, including $\mathrm{HbA} 1 \mathrm{c}$ or oral glucose tolerance tests other than FPG, were not included in the health checkup data of the NHIS-HEALS. Third, the present study adopted the definition of PD if patients had at least two recorded PD diagnoses during outpatient clinic visits or hospitalization with PD diagnosis to reduce the over-estimation of PD $[19,20]$. However, like previous studies using definition of PD based on medical claim codes $[19,20,42,43]$, the possibility of the under- or over-estimation of PD could not be ruled out. Nonetheless, the current study has noteworthy strengths. Particularly, this study was the first to demonstrate the impact of long-term glycemic variability on the development of PD. We used a standardized and validated database created by the Korean government which has a huge sample size and extensive information, including medical diagnoses and medications. In this study, we included only the non-diabetic population to eliminate the possible confounding effects of anti-diabetic medication or diabetic complications, including severe hypoglycemia. In addition, because this study investigated a sample taken from the general population, it could be assumed that the findings reflect the results that exist in the real world. Moreover, we utilized three different indicators, FPG-SD, FPG-CV, and FPG-VIM, to express the variability of glucose and the results were similar and consistent, despite the adjustment for various confounding factors.

\section{Conclusions}

This study demonstrated that visit-to-visit FPG variability in midlife is independently associated with the longterm risk of PD, beyond mean FPG levels and other risk factors. Further research is needed to confirm these

Page 17/23 
findings in other ethnic groups, to discover the pathophysiological mechanisms of glycemic variability leading to the development of PD, and to evaluate the possibility of glycemic variability as a therapeutic target to prevent PD in people with and without diabetes.

\section{Abbreviations}

$A D$, Alzheimer's disease; BMI, body mass index; $C l$, confidence interval; CKD, chronic kidney disease; CV, coefficient of variation; DPP-4, dipeptidyl peptidase-4; FPG, fasting plasma glucose; HbA1c, Hemoglobin A1C; HRs, hazard ratios; ICD-10, International Classification of Diseases Tenth Revision; NHIS-HEALS, National Health Insurance Service-National Health Screening Cohort; PD, Parkinson's disease; SD, standard deviation; VIM, variability independent of the mean

\section{Declarations}

\section{Authors' Contributors}

Study concept and design: HSC, JSL, and KMC; Acquisition of data: HSC, JSL, and KMC; Analysis and interpretation of data: HSC, JSL, JAK, ER, YBL, SH, HJY, JHY, and KMC; Drafting of the manuscript: HSC, JSL, and KMC; Critical revision of the manuscript: NHK, JAS, SGK, NHK, and SHB.

\section{Acknowledgements.}

This study used NHIS data (NHIS-2018-2-107) made by National Health Insurance Service (NHIS). We wish to thank the National Health Insurance Sharing Service (NHISS).

Funding. This study was supported in part by the Korea University Research Fund (K.M.C.) and by the National Research Foundation of Korea (NRF- 2018R1D1A1B07049605, H.S.C.).

\section{Competing interests}

The authors declare that they have no competing interests.

\section{Availability of data and materials}

The datasets used and/or analysed during the present study are available from the corresponding author on reasonable request. 
Not applicable.

Ethics approval

The Korea University institutional review board (IRB) (KUGH17088-001)

Patient consent Not required.

\section{References}

1. Wirdefeldt K, Adami HO, Cole P, Trichopoulos D, Mandel J: Epidemiology and etiology of Parkinson's disease: a review of the evidence. Eur J Epidemiol 2011, 26 Suppl 1:S1-58.

2. Hu G, Jousilahti P, Bidel S, Antikainen R, Tuomilehto J: Type 2 diabetes and the risk of Parkinson's disease. Diabetes Care 2007, 30(4):842-847.

3. Xu Q, Park Y, Huang X, Hollenbeck A, Blair A, Schatzkin A, Chen H: Diabetes and risk of Parkinson's disease. Diabetes Care 2011, 34(4):910-915.

4. Sun Y, Chang YH, Chen HF, Su YH, Su HF, Li CY: Risk of Parkinson disease onset in patients with diabetes: a 9-year population-based cohort study with age and sex stratifications. Diabetes Care 2012, 35(5):1047-1049.

5. Driver JA, Smith A, Buring JE, Gaziano JM, Kurth T, Logroscino G: Prospective cohort study of type 2 diabetes and the risk of Parkinson's disease. Diabetes Care 2008, 31(10):2003-2005.

6. Schernhammer E, Hansen J, Rugbjerg K, Wermuth L, Ritz B: Diabetes and the risk of developing Parkinson's disease in Denmark. Diabetes Care 2011, 34(5):1102-1108.

7. Schwab RS: Progression and prognosis in Parkinson's disease. J Nerv Ment Dis 1960, 130:556-566.

8. Lipman IJ, Boykin ME, Flora RE: Glucose intolerance in Parkinson's disease. J Chronic Dis 1974, 27(1112):573-579.

9. Moran LB, Graeber MB: Towards a pathway definition of Parkinson's disease: a complex disorder with links to cancer, diabetes and inflammation. Neurogenetics 2008, 9(1):1-13.

10. Ceriello A, Esposito K, Piconi L, Ihnat MA, Thorpe JE, Testa R, Boemi M, Giugliano D: Oscillating glucose is more deleterious to endothelial function and oxidative stress than mean glucose in normal and type 2 diabetic patients. Diabetes 2008, 57(5):1349-1354.

11. Monnier L, Mas E, Ginet C, Michel F, Villon L, Cristol JP, Colette C: Activation of oxidative stress by acute glucose fluctuations compared with sustained chronic hyperglycemia in patients with type 2 diabetes. JAMA 2006, 295(14):1681-1687.

12. Gorst C, Kwok CS, Aslam S, Buchan I, Kontopantelis E, Myint PK, Heatlie G, Loke Y, Rutter MK, Mamas MA: Long-term Glycemic Variability and Risk of Adverse Outcomes: A Systematic Review and Meta-analysis. Diabetes Care 2015, 38(12):2354-2369.

13. Yang YF, Li TC, Li Cl, Liu CS, Lin WY, Yang SY, Chiang JH, Huang CC, Sung FC, Lin CC: Visit-to-Visit Glucose Variability Predicts the Development of End-Stage Renal Disease in Type 2 Diabetes: 10-Year Follow-Up of Taiwan Diabetes Study. Medicine (Baltimore) 2015, 94(44):e1804. 
14. Hirakawa Y, Arima H, Zoungas S, Ninomiya T, Cooper M, Hamet P, Mancia G, Poulter N, Harrap S, Woodward $\mathrm{M}$ et al: Impact of visit-to-visit glycemic variability on the risks of macrovascular and microvascular events and all-cause mortality in type 2 diabetes: the ADVANCE trial. Diabetes Care 2014, 37(8):2359-2365.

15. Wang A, Liu X, Xu J, Han X, Su Z, Chen S, Zhang N, Wu S, Wang Y, Wang Y: Visit-to-Visit Variability of Fasting Plasma Glucose and the Risk of Cardiovascular Disease and All-Cause Mortality in the General Population. $J$ Am Heart Assoc 2017, 6(12).

16. Kim JA, Lee JS, Chung HS, Roh E, Lee YB, Hong SH, Kim NH, Yoo HJ, Seo JA, Kim SG et al: Impact of Visit-toVisit Fasting Plasma Glucose Variability on the Development of Type 2 Diabetes: A Nationwide PopulationBased Cohort Study. Diabetes Care 2018, 41(12):2610-2616.

17. Saisho Y: Glycemic variability and oxidative stress: a link between diabetes and cardiovascular disease? Int J Mol Sci 2014, 15(10):18381-18406.

18. Seong SC, Kim YY, Park SK, Khang YH, Kim HC, Park JH, Kang HJ, Do CH, Song JS, Lee EJ et al: Cohort profile: the National Health Insurance Service-National Health Screening Cohort (NHIS-HEALS) in Korea. BMJ Open 2017, 7(9):e016640.

19. Lee SE, Han K, Baek JY, Ko KS, Lee KU, Koh EH, Taskforce Team for Diabetes Fact Sheet of the Korean Diabetes A: Association Between Diabetic Retinopathy and Parkinson Disease: The Korean National Health Insurance Service Database. J Clin Endocrinol Metab 2018, 103(9):3231-3238.

20. Choi HG, Lim JS, Lee YK, Sim S, Kim M: Mortality and cause of death in South Korean patients with Parkinson's disease: a longitudinal follow-up study using a national sample cohort. BMJ Open 2019, 9(9):e029776.

21. Lin DY, Wei LJ, Ying Z: Checking the Cox Model with Cumulative Sums of Martingale-Based Residuals. Biometrika 1993, 80(3):557-572.

22. Whitmer RA, Karter AJ, Yaffe K, Quesenberry CP, Jr., Selby JV: Hypoglycemic episodes and risk of dementia in older patients with type 2 diabetes mellitus. JAMA 2009, 301(15):1565-1572.

23. Rawlings AM, Sharrett AR, Mosley TH, Ballew SH, Deal JA, Selvin E: Glucose Peaks and the Risk of Dementia and 20-Year Cognitive Decline. Diabetes Care 2017, 40(7):879-886.

24. Li TC, Yang CP, Tseng ST, Li Cl, Liu CS, Lin WY, Hwang KL, Yang SY, Chiang JH, Lin CC: Visit-to-Visit Variations in Fasting Plasma Glucose and HbA1c Associated With an Increased Risk of Alzheimer Disease: Taiwan Diabetes Study. Diabetes Care 2017, 40(9):1210-1217.

25. Lin CC, Yang CP, Li Cl, Liu CS, Chen CC, Lin WY, Hwang KL, Yang SY, Li TC: Visit-to-visit variability of fasting plasma glucose as predictor of ischemic stroke: competing risk analysis in a national cohort of Taiwan Diabetes Study. BMC Med 2014, 12:165.

26. Bancks MP, Carnethon MR, Jacobs DR, Jr., Launer LJ, Reis JP, Schreiner PJ, Shah RV, Sidney S, Yaffe K, Yano $Y$ et al: Fasting Glucose Variability in Young Adulthood and Cognitive Function in Middle Age: The Coronary Artery Risk Development in Young Adults (CARDIA) Study. Diabetes Care 2018, 41(12):2579-2585.

27. Arvanitakis Z, Wilson RS, Bienias JL, Bennett DA: Diabetes and parkinsonian signs in older persons. Alzheimer Dis Assoc Disord 2007, 21(2):144-149.

28. Umeno A, Biju V, Yoshida Y: In vivo ROS production and use of oxidative stress-derived biomarkers to detect the onset of diseases such as Alzheimer's disease, Parkinson's disease, and diabetes. Free Radic Res 2017, 51(4):413-427. 
29. Cadet JL, Brannock C: Free radicals and the pathobiology of brain dopamine systems. Neurochem Int 1998, 32(2):117-131.

30. Esposito K, Nappo F, Marfella R, Giugliano G, Giugliano F, Ciotola M, Quagliaro L, Ceriello A, Giugliano D: Inflammatory cytokine concentrations are acutely increased by hyperglycemia in humans: role of oxidative stress. Circulation 2002, 106(16):2067-2072.

31. Arnold SE, Arvanitakis Z, Macauley-Rambach SL, Koenig AM, Wang HY, Ahima RS, Craft S, Gandy S, Buettner C, Stoeckel LE et al: Brain insulin resistance in type 2 diabetes and Alzheimer disease: concepts and conundrums. Nat Rev Neurol 2018, 14(3):168-181.

32. Stout RW: Insulin and atheroma. 20-yr perspective. Diabetes Care 1990, 13(6):631-654.

33. Athauda D, Foltynie T: Insulin resistance and Parkinson's disease: A new target for disease modification? Prog Neurobiol 2016, 145-146:98-120.

34. Bassil F, Fernagut PO, Bezard E, Meissner WG: Insulin, IGF-1 and GLP-1 signaling in neurodegenerative disorders: targets for disease modification? Prog Neurobio/ 2014, 118:1-18.

35. Takahashi M, Yamada T, Tooyama I, Moroo I, Kimura H, Yamamoto T, Okada H: Insulin receptor mRNA in the substantia nigra in Parkinson's disease. Neurosci Lett 1996, 204(3):201-204.

36. Kim SR, Ries V, Cheng HC, Kareva T, Oo TF, Yu WH, Duff K, Kholodilov N, Burke RE: Age and alpha-synuclein expression interact to reveal a dependence of dopaminergic axons on endogenous Akt/PKB signaling. Neurobiol Dis 2011, 44(2):215-222.

37. De Felice FG, Lourenco MV, Ferreira ST: How does brain insulin resistance develop in Alzheimer's disease? Alzheimers Dement 2014, 10(1 Suppl):S26-32.

38. Ferreira ST, Clarke JR, Bomfim TR, De Felice FG: Inflammation, defective insulin signaling, and neuronal dysfunction in Alzheimer's disease. Alzheimers Dement 2014, 10(1 Suppl):S76-83.

39. Green H, Tsitsi P, Markaki I, Aarsland D, Svenningsson P: Novel Treatment Opportunities Against Cognitive Impairment in Parkinson's Disease with an Emphasis on Diabetes-Related Pathways. CNS Drugs 2019, 33(2):143-160.

40. Park SE, Lee BW, Kim JH, Lee WJ, Cho JH, Jung CH, Lee SH, Suh S, Hur GC, Kim SH et al: Effect of gemigliptin on glycaemic variability in patients with type 2 diabetes (STABLE study). Diabetes Obes Metab 2017, 19(6):892-896.

41. Svenningsson P, Wirdefeldt K, Yin L, Fang F, Markaki I, Efendic S, Ludvigsson JF: Reduced incidence of Parkinson's disease after dipeptidyl peptidase-4 inhibitors-A nationwide case-control study. Mov Disord 2016, 31(9):1422-1423.

42. Lee H, Myung W, Kim DK, Kim SE, Kim CT, Kim H: Short-term air pollution exposure aggravates Parkinson's disease in a population-based cohort. Sci Rep 2017, 7:44741.

43. Moon JY, Kim HJ, Park YH, Park TK, Park EC, Kim CY, Lee SH: Association between Open-Angle Glaucoma and the Risks of Alzheimer's and Parkinson's Diseases in South Korea: A 10-year Nationwide Cohort Study. Sci Rep 2018, 8(1):11161.

\section{Figures}


Fig 1.

(A)

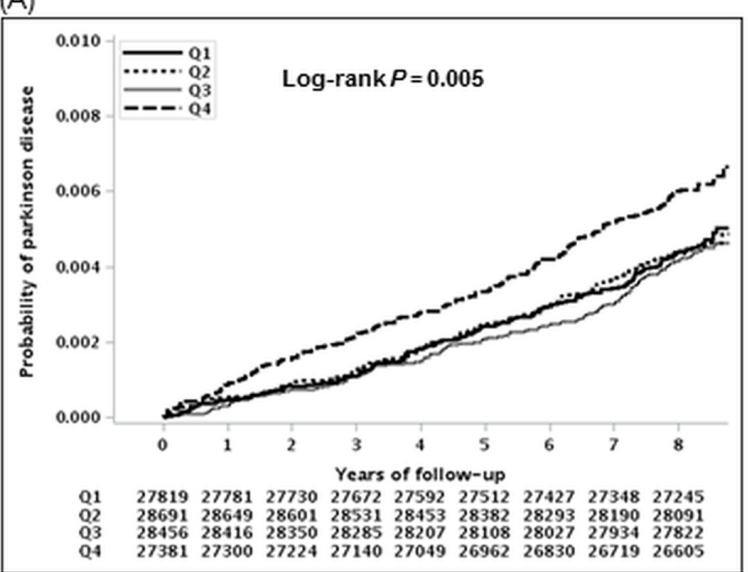

(B)

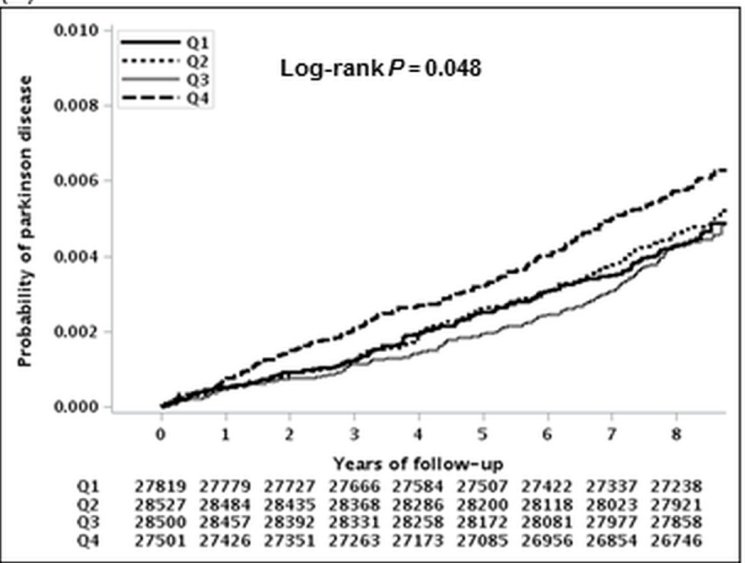

(C)

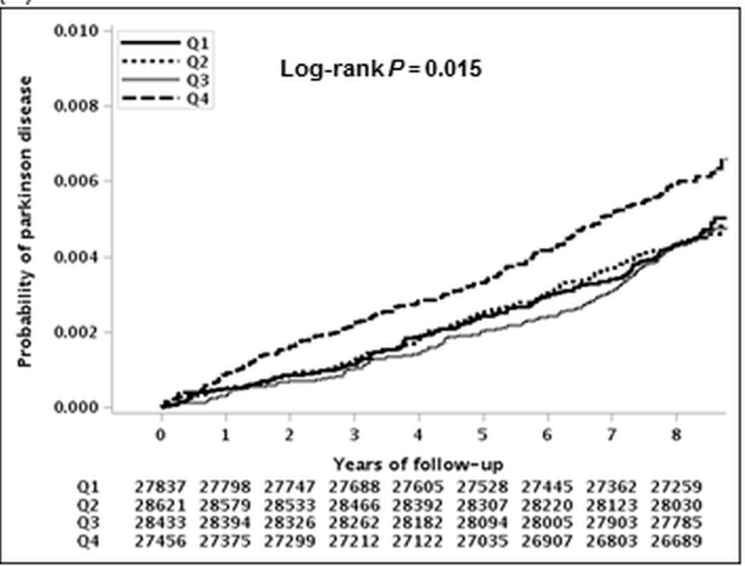

Figure 1

Kaplan-Meier estimates of probability of Parkinson's disease (PD) expressed as quartiles of fasting plasma glucose (FPG) variability in the midlife group (A) FPG variability (FPG -SD: standard deviation) (B) FPG variability (FPG - CV: coefficient of variance) (C) FPG variability (FPG - VIM: variability independent of the mean)

\section{Supplementary Files}

This is a list of supplementary files associated with this preprint. Click to download. 
- SupplementalMaterialvFPGPD.docx

- STROBEChecklist.docx 